\title{
Conf- $950226-.52$
}

UCRL-JC-120009

PREPRINT

\section{Development of High Damage Threshold Optics for Petawatt-Class Short-Pulse Lasers}

\author{
B.C. Stuart \\ M. D. Perry \\ R. D. Boyd \\ J. A. Britten \\ B. W. Shore \\ M. D. Feit
}

A. M. Rubenchik

This paper was prepared for submittal to the

Lasers and Applications Conference

San Jose, CA

February 4-10, 1995

February 22, 1995

This is a preprint of a paper intended for publication in a journal or proceedings. Since changes may be made before publication, this preprint is made available with the understanding that it will not be cited or reproduced without the permission of the author. 


\section{DISCLAIMER}

This document was prepared as an account of work sponsored by an agency of the United States Government. Neither the United States Government nor the University of California nor any of their employees, makes any warranty, express or implied, or assumes any legal liability or responsibility for the accuracy, completeness, or usefuiness of any information, apparatus, product, or process disclosed, or represents that its use would not infringe privately owned rights. Reference herein to any specific commercial product, process, or service by trade name, trademark, manufacturer, or otherwise, does not necessarily constitute or imply its endorsement, recommendation, or favoring by the United States Government or the University of California. The views and opinions of authors expressed herein do not necessarily state or reflect those of the United States Government or the University of California, and shall not be used for advertising or product endorsement purposes. 


\section{DISCLAIMER}

Portions of this document may be illegible in electronic image products. Images are produced from the best available original document. 
Development of high damage threshold optics for petawatt-class short-pulse lasers

\author{
B.C. Stuart, M.D. Perry, R.D. Boyd, J.A. Britten, B.W. Shore, M.D. Feit, A.M. Rubenchik \\ Lawrence Livermore National Laboratory \\ P.O. Box 808, L-493, Livermore, CA 94550
}

\begin{abstract}
We report laser-induced damage threshold measurements on pure and multilayer dielectrics and goldcoated optics at 1053 and $526 \mathrm{~nm}$ for pulse durations, $\tau$, ranging from $140 \mathrm{fs}$ to $1 \mathrm{~ns}$. Damage thresholds of gold coatings are limited to $500 \mathrm{~mJ} / \mathrm{cm}^{2}$ in the subpicosecond range for $1053-\mathrm{nm}$ pulses. In dielectrics, qualitative differences in the morphology of damage and a departure from the diffusion-dominated $\tau 1 / 2$ scaling indicate that damage results from plasma formation and ablation for $\tau \leq 10 \mathrm{ps}$ and from conventional melting and boiling for $\tau>50 \mathrm{ps}$. A theoretical model based on electron production via multiphoton ionization, Joule heating, and collisional (avalanche) ionization is in quantitative agreement with both the pulsewidth and wavelength scaling of experimental results.
\end{abstract}

Keywords: short-pulse, laser-induced damage, dielectric breakdown, multiphoton ionization

\title{
1. INTRODUCTION
}

The application of chirped-pulse amplification 1 (CPA) to broadband, high energy solid-state lasers has enabled terawatt class systems producing subpicosecond pulses. Further increase in the peak power available from such systems ${ }^{2}$ is now limited by damage to optical surfaces due to the intense short pulses. The subpicosecond pulse duration is significantly shorter than the time scale for electron energy transfer to the lattice. As a result, damage caused by subpicosecond pulses is characterized by ablation, with essentially no collateral damage. Many applications, ranging from materials processing to biomedical technologies, could potentially benefit from the more localized energy deposition with these short pulses.

The scaling relationship of the damage threshold as a function of pulsewidth is of interest for all optical components in a high-power laser system: mirrors, polarizers, bulk dielectrics, coatings, and diffraction gratings. At this time, the gratings used for pulse compression typically exhibit the lowest damage threshold of any component. These high-efficiency diffraction gratings have traditionally been based on metallic gratings. High-efficiency multilayer dielectric diffraction gratings ${ }^{3}$ offer a promising alternative that should exhibit a damage threshold at least three times that of gold gratings. In this paper we present measurements and theoretical analysis of laser-induced damage to gold-coated, multilayer dielectric, and pure dielectric optical components.

Investigation of the pulsewidth dependence of laser-induced damage to dielectrics has been the subject of numerous studies ${ }^{4-25}$. For pulses longer than a few tens of picoseconds, the generally accepted picture of bulk damage to defect-free dielectrics involves the heating of conduction band electrons by the incident radiation and transfer of this energy to the lattice. Damage occurs via conventional heat deposition resulting in melting and boiling of the dielectric material. Because the controlling rate is that of thermal conduction through the lattice, this model predicts ${ }^{13}$ a $\tau^{1 / 2}$ dependence of the threshold fluence upon pulse duration $\tau$. This is in reasonably good agreement with numerous experiments $16-25$ which have 
observed a $\tau \alpha$ scaling with nominally $0.3<\alpha<0.6$ in a variety of dielectric materials (including samples with defects) from 20 ps to over $100 \mathrm{~ns}$.

Here, we report measurements of damage thresholds for fused silica, calcium fluoride, and multilayer dielectrics for pulses ranging from $140 \mathrm{fs}$ to $1 \mathrm{~ns}$. In each of these large-bandgap materials we observe a change in the damage mechanism and morphology for pulses shorter than $20 \mathrm{ps.} \mathrm{Although} \mathrm{we}$ observe a strong deviation from the $\tau 1 / 2$ scaling, we find no evidence for an increase in damage threshold with decreasing pulsewidth as reported by $\mathrm{Du}$ et al 24 . Instead, we observe a decreasing threshold associated with a gradual transition from the long-pulse, thermally-dominated regime to an ablative regime dominated by collisional and multiphoton ionization, and plasma formation. A general theoretical model 25 of laser interaction with dielectrics, based on multiphoton ionization, Joule heating, and collisional (avalanche) ionization, is shown to be in good agreement with the data in this short-pulse regime.

\section{DAMAGE THRESHOLD MEASUREMENT}

For damage testing, we used laser pulses generated by a 1053-nm Ti:sapphire CPA system 26 . The front-end of this system produced 1-ns stretched pulses of up to $60 \mathrm{~mJ}$ at $10 \mathrm{~Hz}$. The pulses were compressed in a four-pass, single-grating compressor of variable length (Figure 1). By varying the dispersive path length of the compressor, we obtained pulses of continuously adjustable duration from 0.3 to 1 ns. Pulse durations were measured with a single-shot autocorrelator (0.3-1.5 ps), streak camera (10$1000 \mathrm{ps})$, and fast photodiode (100-1000 ps), and calibrated against the linear position of the fold mirrors. The temporal profile of the compressed pulses depends strongly on the spectral and temporal profile of the stretched pulse. Pulse compression with spectral clipping is analogous to diffraction from a hard-edge aperture in the spatial domain, and results in a modulated temporal profile in the "intermediate" range of compression. For these damage measurements, we compressed a near-Gaussian spectral profile to obtain temporally smooth output pulses. This allowed us to easily relate the time evolution of the pulse intensity to the measured fluence.

We also measured damage thresholds with 526-nm light generated by frequency-doubling the 1053-nm compressed pulses in a thin (4-mm) potassium dideuterium phosphate $\left(\mathrm{KD}^{*} \mathrm{P}\right)$ crystal. The conversion efficiency was kept below $25 \%$ to avoid any temporal distortion of the second-harmonic pulse. We measured our shortest 526-nm pulses with a single-shot autocorrelator to be $275 \mathrm{fs}$. This was in good agreement with the expected $2^{1 / 2}$ scaling from the $1053-\mathrm{nm}$ pulsewidth (415 fs), so this scaling was used for the other 526-nm pulsewidths. Data was also taken at $825 \mathrm{~nm}$ and $140 \mathrm{fs}$ with a Cr:LiSAF CPA system 27 to confirm the decreasing trend in damage fluence with pulsewidth.

The energy of each pulse was monitored with the leakage through a $92 \%$ reflectivity mirror. We adjusted the energy delivered to the damage sample with a half-waveplate before compression, using the strong dependence of grating efficiency upon input polarization. The rms energy stability was typically $1.5 \%$, and we report the average value here. We performed damage measurements with laser spot sizes adjustable from 0.3 to $1.0-\mathrm{mm}$ diameter ( $\mathrm{e}^{-2}$ intensity). Laser pulses were focused onto the damage sample by a $1-\mathrm{m}$ focal length lens, with a variable distance to the sample. The spot size was measured on a CCD camera. With the shortest pulses we used, the intensity (up to $4 \times 10^{12} \mathrm{~W} / \mathrm{cm}^{2}$ on sample) became high enough to cause significant ( $10 \%$ effect) whole-beam self-focusing in the focusing lens and the air path leading to the sample. All beam size measurements were therefore performed with a 4:1 image of the 
beam taken from a $4 \%$ reflection at the position of the damage sample, and at or just below damage threshold. The laser mode at the sample had a $98 \%$ or better fit to a Gaussian, so the effective diameter as measured on the camera system was combined with the measured energy to give the pulse energy fluence. Our estimated absolute uncertainty in fluence was $15 \%$, but relative values should be within $5 \%$.

After irradiation, Nomarski microscopy was used to inspect the sample for possible damage. We define damage to be any visible permanent modification to the surface observable with the Nomarski microscope. The smallest damage spots we could observe were approximately $0.5 \mu \mathrm{m}$ in diameter, a factor

of $10^{6}$ smaller in area than the laser spot size and nearly impossible to observe by other methods (e.g., degradation of transmission, scattered light, etc.). To avoid the complications of spatial and temporal distortion caused by self-focusing, group velocity dispersion, and self-phase modulation, we considered only front-surface damage.

Initial damage, at threshold, may have many forms: ablation of a very small amount of material (a few atomic layers); formation of a color center, shallow traps, or lattice defects; or melting of a very small volume. These weak effects are very difficult to detect. In order to "amplify" this damage to an easily observable size, and to minimize statistical uncertainty, we conducted our damage testing with multiple pulses of a given fluence on each site ( $\mathrm{S}: 1)$. This is in contrast to the single-shot measurements of Du $e t$ $a^{24}$, where their diagnostics of pulse transmission and plasma emission require a macroscopic damage site on a single pulse. We typically used six hundred shots at $10 \mathrm{~Hz}$, unless damage was obvious sooner. Many fluence levels (15-30) were examined above and below the damage threshold for a given pulsewidth in order to establish the threshold value.

\section{DAMAGE RESULTS}

\subsection{Gold-coated optics}

For use with high-power lasers, the damage threshold of a grating (or mirror) has an importance equal to the diffraction efficiency (reflectivity). We have achieved diffraction efficiencies greater than $95 \%$ for 1710 line $/ \mathrm{mm}$ gold-coated gratings at $1064 \mathrm{~nm}$ and efficiencies greater than $94 \%$ at $830 \mathrm{~nm}$. This efficiency has been achieved on both small scale $(<15 \mathrm{~cm})$ and large scale $(30 \mathrm{~cm}$ diameter) diffraction gratings. ${ }^{28}$ In pulse compression, the damage threshold limits the amount of energy which can be tolerated in the pulse for a given grating area. The low damage threshold of diffraction gratings is also responsible for their limitation to use in low power tunable oscillators. Narrow linewidth, grating cavity systems based on broadband solid-state materials such as Ti:Sapphire, Alexandrite and Cr:LiSAF require gratings exhibiting damage thresholds in excess of $2 \mathrm{~J} / \mathrm{cm}^{2}$ in order to access the high energy storage of these materials.

We have made a systematic study of the relationship between gold-coating thickness and laser damage threshold. We find thickness to be the dominant factor in determining the damage threshold of defect-free metallic gratings. With the exception of gratings ruled directly into the metal, all metal gratings are formed by vapor deposition of a thin metal film onto a dielectric surface. The dielectric may be photoresist, epoxy, or glass. All of these materials exhibit low thermal conductivity relative to the metal film, and so they essentially serve as a thermal barrier. 


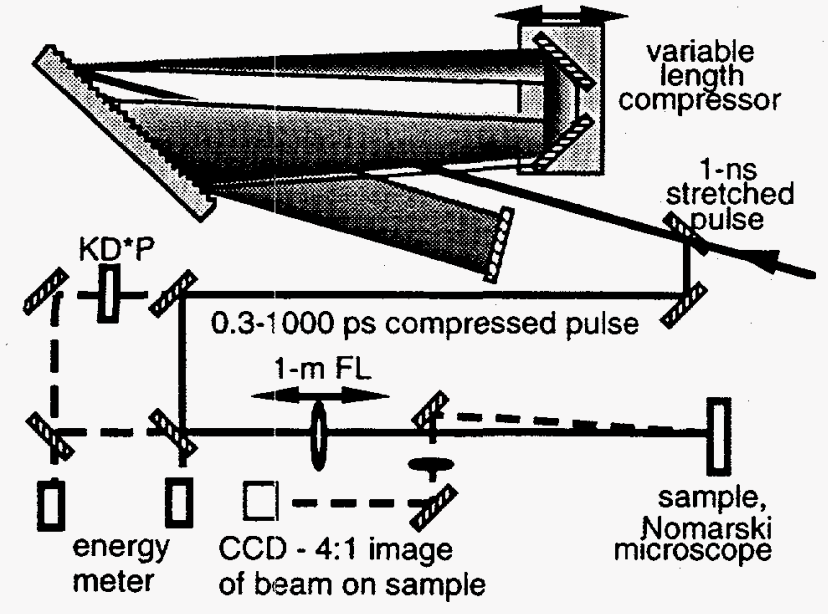

Figure 1. The 1-ns stretched pulses are compressed to 0.31000 ps by a variable length compressor. A $1-\mathrm{m}$ focal length lens focuses the pulses on the sample and the spot size is measured with a $4: 1$ image.

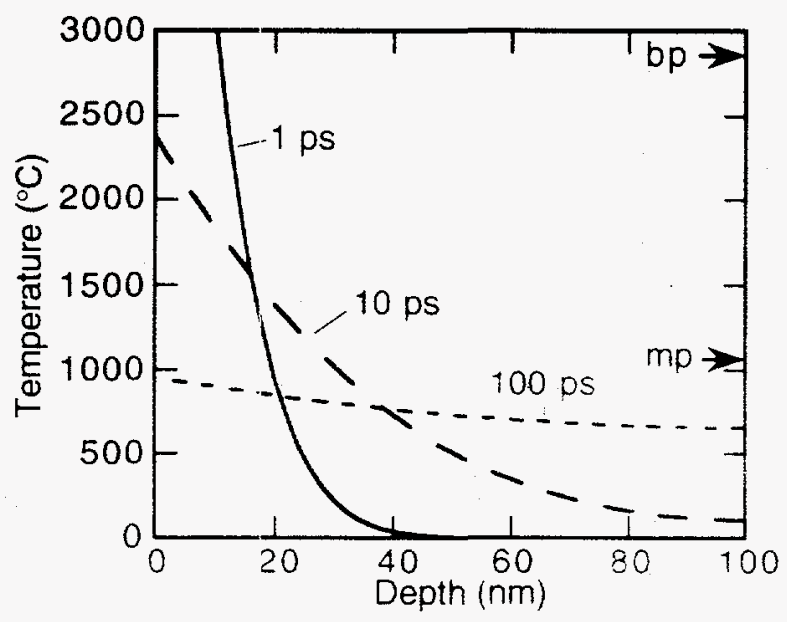

Figure 3. Temperature distribution as a function of depth and pulse duration for an absorbed fluence of $500 \mathrm{~mJ} / \mathrm{cm}^{2}$ delivered to a $100-\mathrm{nm}$ thick gold film. The melting $(\mathrm{mp})$ and boiling (bp) temperatures for gold are indicated
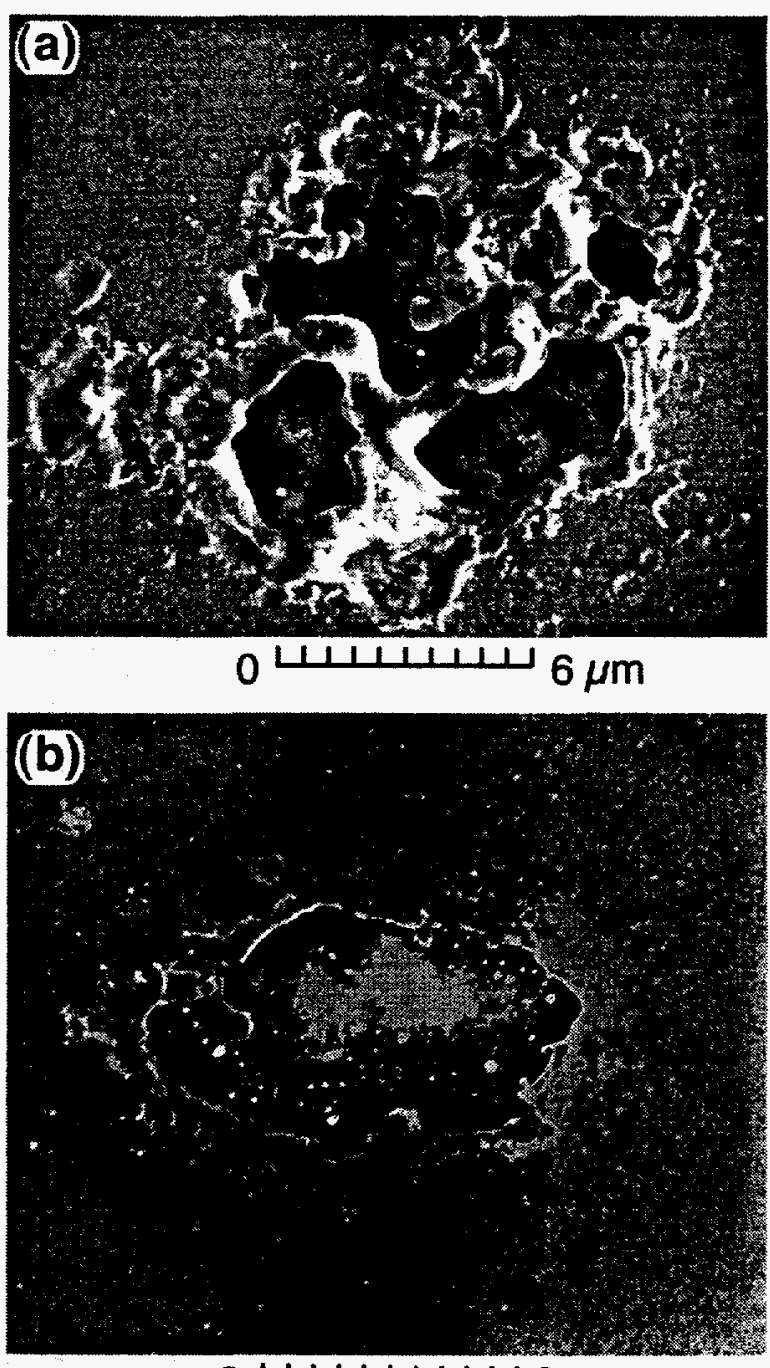

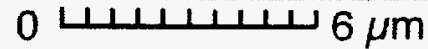

Figure 2. Damage to gold film with 1053-nm pulses: (a) long-pulse. $900 \mathrm{ps}$, (b) short-pulse. $0.6 \mathrm{ps}$.

Damage is more readily produced by short pulses than by long ones. Scanning microscopy of the damaged regions of the surface of a plain gold film reveais strong clues : : mechanism responsible for damage. The damage spots created by very short pules exhit neghat melting or collateral damage (Figure 2a) indicative of exremely rapid ablation and iaporizatum.; contrast, longer pulses create larger damage regions whow apnearance is consistent wh melting, itow and subsequent resolidification (Figure 2b). These onscrvitions form the basis of at simple model for lase: damage to gold-coated optics 28 . Specifically w a wume that damage occurs when the temperature reaches the melting point of the metal for long pules or the boiling point for short pulses. We model the grating as a one dimensional film suhiected to a constant heat flux at the surface (provided by the laser 
pulse) and permit negligible heat transfer at the interface between the dielectric and the metal. Laser energy is deposited in a skin depth, which for gold at a laser wavelength of $1 \mu \mathrm{m}$, is only $3 \mathrm{~nm}$. As a result, we approximate the boundary condition at the surface of the grating as one of a constant heat flux deposited at the surface. The dependence on grating parameters such as groove spacing, depth and shape, can be accounted for by adjusting the absorption coefficient.

Starting with a gold film at a uniform temperature, Figure 3 shows the temperature distribution in a 100 -nm thick gold coating immediately following irradiation by $1053-\mathrm{nm}$ laser pulses of various durations at a fluence of $500 \mathrm{~mJ} / \mathrm{cm}^{2}$. For subpicosecond pulses, a depth of less than $10 \mathrm{~nm}$ is heated beyond the boiling point, and little heat transfers into the remaining film. This is a result of the finite thermal diffusivity of gold, $\alpha=143 \mathrm{~nm}^{2} / \mathrm{psec}$. For such short picosecond pulses the thermal wave cannot penetrate more than approximately $10 \mathrm{~nm}\left(\alpha \mathrm{t} / \mathrm{L}^{2} \approx 1\right)$. Hence, we would predict that at this fluence, the surface will be damaged, regardless of the thickness of the coating. For longer pulses the coating thickness has a significant effect on the surface temperature and the resulting damage threshold. The thermal diffusivity is sufficiently high that the metal can efficiently conduct heat away from the surface in a few picoseconds. Thus, for pulses which deposit their energy during a few tens of picoseconds, the surface temperature is depressed by conduction throughout the bulk of the film. For pulses longer than $100 \mathrm{ps,}$ conduction across the film is complete and the temperature becomes constant throughout the film. In this case the bulk temperature, and hence the damage threshold, is determined solely by the thickness of the coating (i.e. the volume of material heated).

Defining the damage threshold as the onset of melting for long pulses and boiling for short pulses, we use this one-dimensional heat-conduction model to predict the dependence of damage threshold on coating thickness and laser pulse width. In Figure 4 we show our calculated and measured damage thresholds for 1053-nm laser pulses incident on a gold film deposited on developed photoresist. For (long) nanosecond pulses, there is an approximately linear dependence on film thickness up to $200 \mathrm{~nm}$, followed by an asymptotic approach to the damage threshold of bulk material. For (short) picosecond pulses, we observe a dependence on film thickness similar to that of nanosecond pulses, but only to a thickness near the penetration depth. Beyond this thickness, the damage threshold is predicted to be independent of film thickness. The difference between the model and the measured data for picosecond pulses for coatings below $100 \mathrm{~nm}$ in thickness is due to pinholes and defects in films less than $100 \mathrm{~nm}$. As the coating thickness increased above $100 \mathrm{~nm}$, the coatings became more uniform and increasingly defect free.

The measured damage thresholds of some of our high efficiency gold gratings are also shown in Figure 4. Although the damage threshold of the gratings is always lower than that observed for uniform metal films, we observe the same general dependence on coating thickness up to approximately $200 \mathrm{~nm}$. We expect that gold-coated gratings, when free of surface defects, should have damage thresholds approaching those of gold films. The calculations shown in Figure 4 are applicable to high efficiency gratings where the absorption is determined only by the bulk material properties. In those cases where trapped fields are present 29 , the absorption coefficient is dominated by the grating structure, and the damage threshold is significantly lower. We do not account for the possibility of field enhancement and resulting plasma formation. Field enhancement is a weak effect in the high efficiency, near sinusoidal gratings considered here. Furthermore, plasma formation will be dominant only for very short pulses (less than $20 \mathrm{fs}$ ) where the effective irradiance is on the order of $10^{13} \mathrm{~W} / \mathrm{cm}^{2}$. 
In addition to testing our own gratings, we have tested, at $1053 \mathrm{~nm}$, the laser damage threshold of samples of several commercially available gold coated diffraction gratings. For nanosecond pulses, the damage thresholds have varied widely from as low as $50 \mathrm{~mJ} / \mathrm{cm}^{2}$ to over $400 \mathrm{~mJ} / \mathrm{cm}^{2}$ for holographic gratings, and up to $800 \mathrm{~mJ} / \mathrm{cm}^{2}$ for gold coated ruled gratings. Our tests showed little correlation between diffraction efficiency and damage threshold. The commercial metallic gratings varied dramatically in composition, ranging from holographic masters in which the metal film is deposited directly on the developed photoresist to replica gratings where the metal is deposited on an epoxy. Surface preparation, groove shape and coating thickness all varied greatly between samples from different suppliers.

\subsection{Fused silica}

Since gold-coated optics cannot withstand fluences much greater than $500 \mathrm{~mJ} / \mathrm{cm}^{2}$ in the shortpulse (subpicosecond) regime, we turned to multilayer dielectrics to create the high damage threshold gratings 3 necessary for pulse compression of high-energy $(\mathrm{kJ})$ laser pulses. We began with the desire to understand the physical damage mechanism in dielectrics, and chose fused silica as our first sample due to its availability in high-purity samples and its extensive physical characterization in the literature.

The results presented here were obtained with $1-\mathrm{cm}$ thick "super-polished" fused silica samples (Corning 7940) exhibiting less than 1-nm rms surface roughness. We measured the same damage thresholds with a $200-\mu \mathrm{m}$ thick fused-silica etalon, which was tested to examine any possible differences between thick and thin samples. Some samples were cleaned initially with acetone or methanol, and all were cleaned when damage debris accumulated on the surface. No difference in threshold was found between samples or areas on a given sample that were or were not cleaned. Defects visible through the microscope were avoided. With short $(0.4 \mathrm{ps})$ pulses, damage always occurred at the location corresponding to the peak of the Gaussian intensity profile, indicating that defect sites did not contribute to our measured thresholds. Ramping the fluence with short pulses, which would ionize and ablate any low-

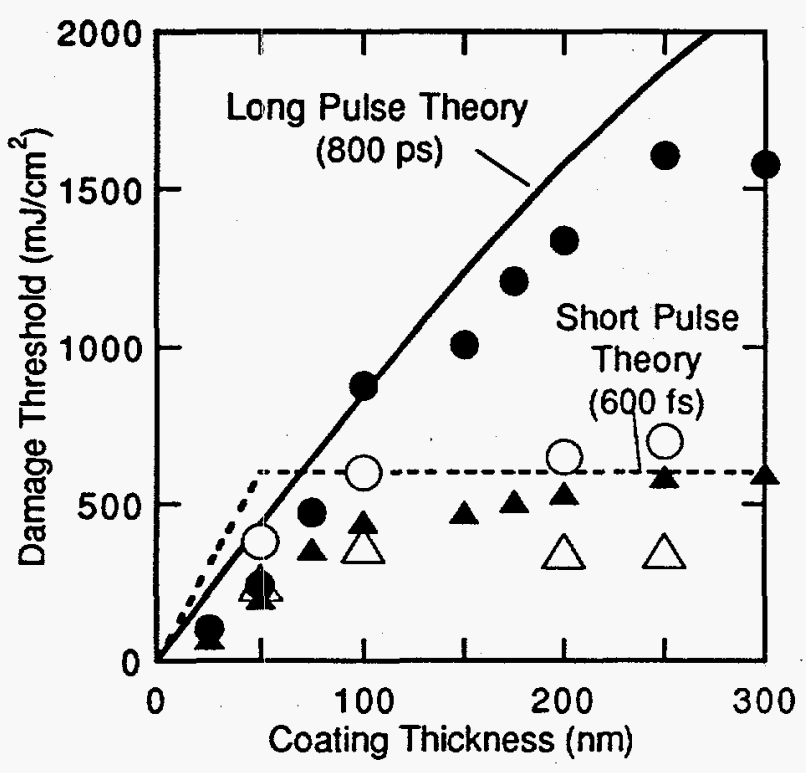

Figure 4.Predicted and measured 1053-nm damage thresholds for gold films. Filled symbols show measured values for gold deposited on photoresist, open symbols show measured values for gold gratings. Circles are long-pulse (800 ps), triangles are short-pulse $(600 \mathrm{fs})$. Lines show theoretical predictions.

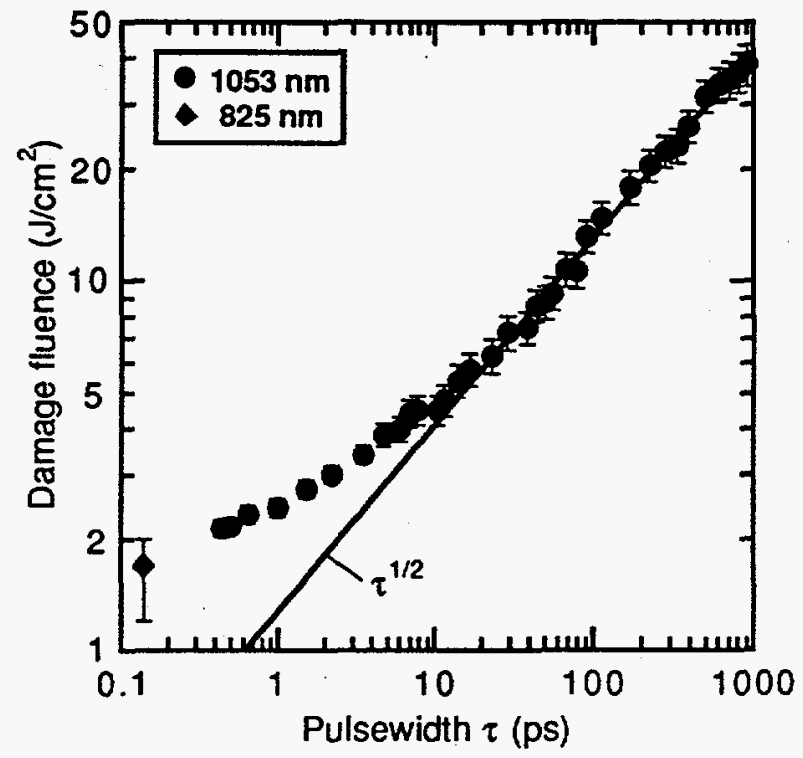

Figure 5. Pulsewidth dependence of threshold damage fluence for fused silica 
lying states or surface contamination with lower threshold, gave the same threshold as our measurements with constant fluence. We thus believe that our measurements correspond to a uniform, defect-free surface and can be compared to calculations based on the intrinsic properties of fused silica.

Our measured threshold damage fluence for fused silica at $1053 \mathrm{~nm}$ as a function of laser pulse length (FWHM) is shown in Figure 5. In the long-pulse regime ( $\tau>20 \mathrm{ps)}$, the data fit well to a $\tau^{1 / 2}$ dependence (actual fit: $\tau^{0.504}$ ), characteristic of transfer of electron kinetic energy to the lattice and diffusion during the laser pulse. The damage occurs over the entire area irradiated as shown in the electron micrograph of Figure 6(a) (All damage micrographs shown are the result of multiple pulses). The damage is thermal in nature and characterized by melting and boiling of the surface. This is more easily seen in Figure 7(a), which shows the edge of the long-pulse damage spot. For pulses shorter than $20 \mathrm{ps,} \mathrm{the}$ damage fluence no longer follows the $\tau^{1 / 2}$ dependence and exhibits a morphology dramatically different from that observed with long pulses. Short-pulse damage is confined to a small region at the peak of the Gaussian irradiance distribution (Figure 6(b)). Damage occurs only over an area with sufficient intensity to
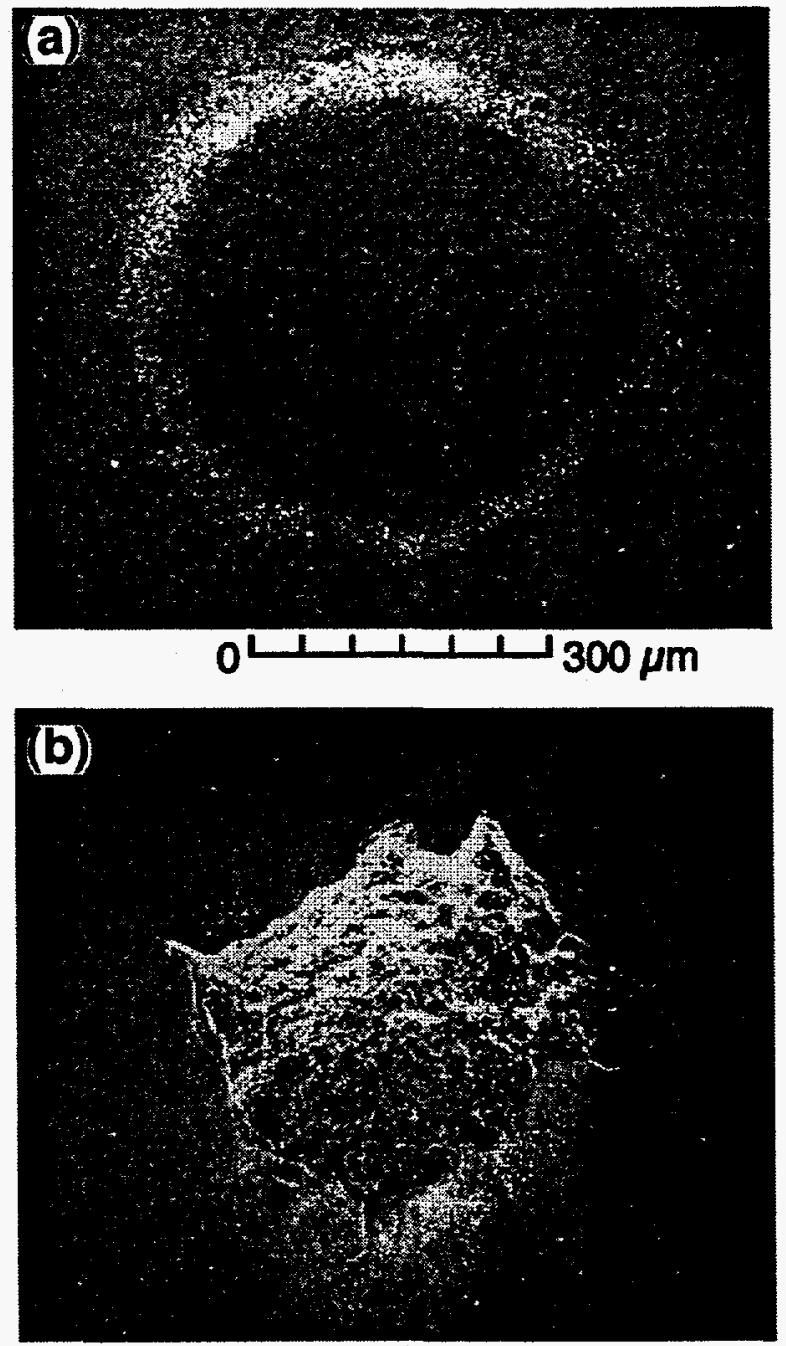

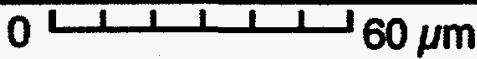

Figure 6. Laser damage spots on fused silica created by: (a) long-pulse, 900-ps, $300 \mu \mathrm{m}$ diameter, (b) short-pulse, 0.4ps, 500- $\mu \mathrm{m}$ diameter.
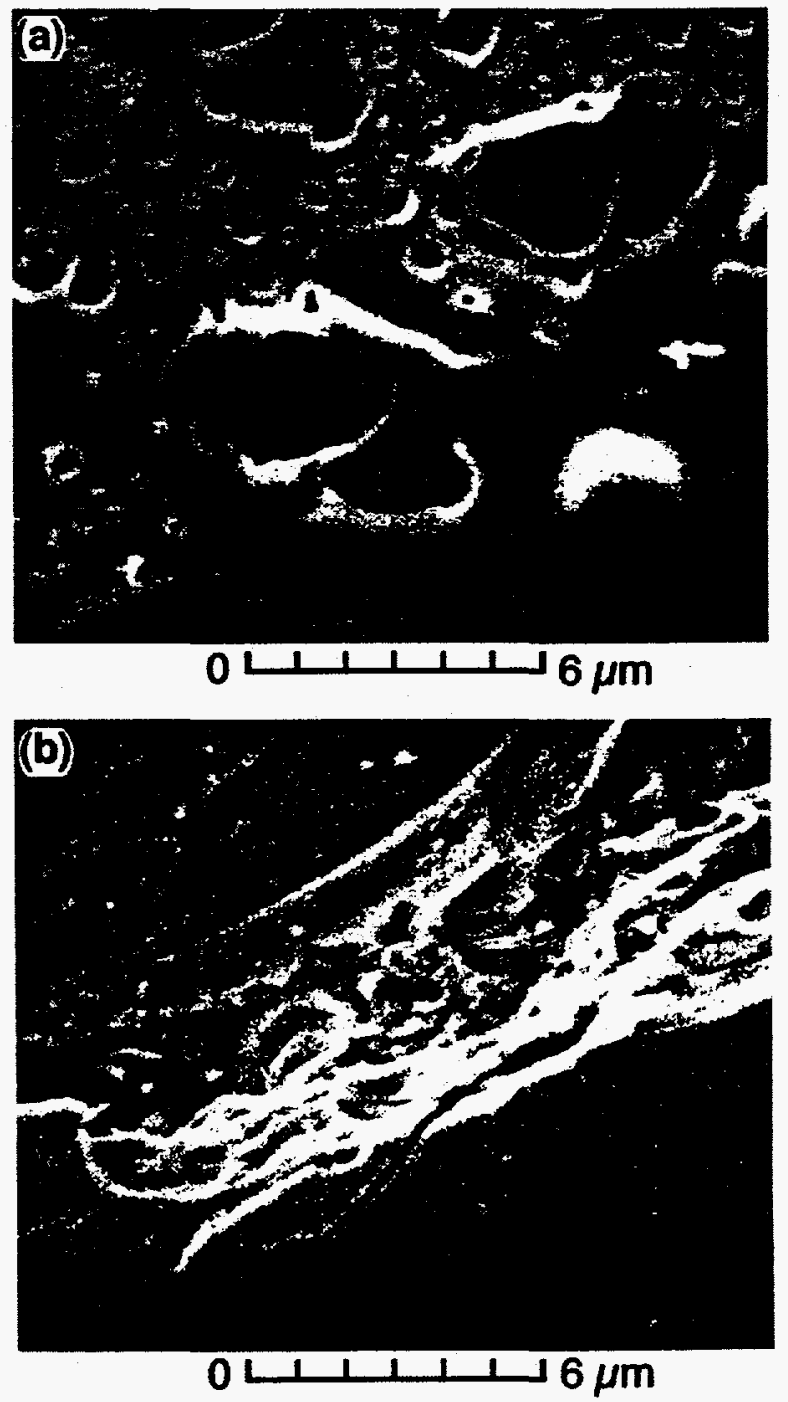

Figure 7 .Edges of laser damage spots of Fig. 6: (a) long pulse, 900 ps, (b) short-pulse, 0.4 ps. 
produce ionization. With insufficient time for lattice coupling, there is no collateral damage. As a result, the damaged area can be many orders of magnitude smaller with short $(\tau<10 \mathrm{ps})$ pulses than with long pulses. For the case of fused silica shown in Figure 6, the damaged area produced by the $0.5-\mathrm{mm}$ diameter, 500 -fs pulse was two orders of magnitude smaller than that produced by the 0.3 -mm diameter, 900-ps pulse. Short-pulse damage appears as a shallow fractured and pitted crater characteristic of a thin layer of material removed by ablation (Figure 7(b)). We found damage in the short-pulse limit to be deterministic, with only a couple percent fluence range between damage and no damage. Fused silica irradiated with 10000 shots at $2 \%$ below our determined threshold showed no evidence of damage with 0.4-ps pulses. For long pulses we found a roughly $10 \%$ range in fluence where damage would or would not occur.

Optical breakdown in transparent materials can be understood in terms of an electron avalanche $5,6,12,14,15$ in which conduction-band electrons, oscillating in response to the laser field, transfer energy by scattering from phonons. If an electron can achieve an energy equal to the bandgap, subsequent impact ionization promotes another valence electron into the conduction band. The resulting avalanche, similar to that in gases 30 , leads to an irreversible change in the bulk structure. We have developed 25 a general theoretical model of laser interaction with dielectrics in which very short intense pulses produce initial conduction band electrons by multiphoton ionization. Because the pulses are so short, collisional heating of the electrons occurs before there is significant transfer of energy from the electrons to the lattice. This heating and energy diffusion, combined with impact ionization, result in an electron avalanche which can be described by a kinetic equation for the electron distribution function.

Figure 8 illustrates the calculated evolution of electron density for a $100-\mathrm{fs}, 12-\mathrm{TW} / \mathrm{cm}^{2}$ pulse. The pulse intensity and the electron density produced by photoionization alone are included for reference. Because photoionization is extremely intensity dependent, the electron production takes place principally at

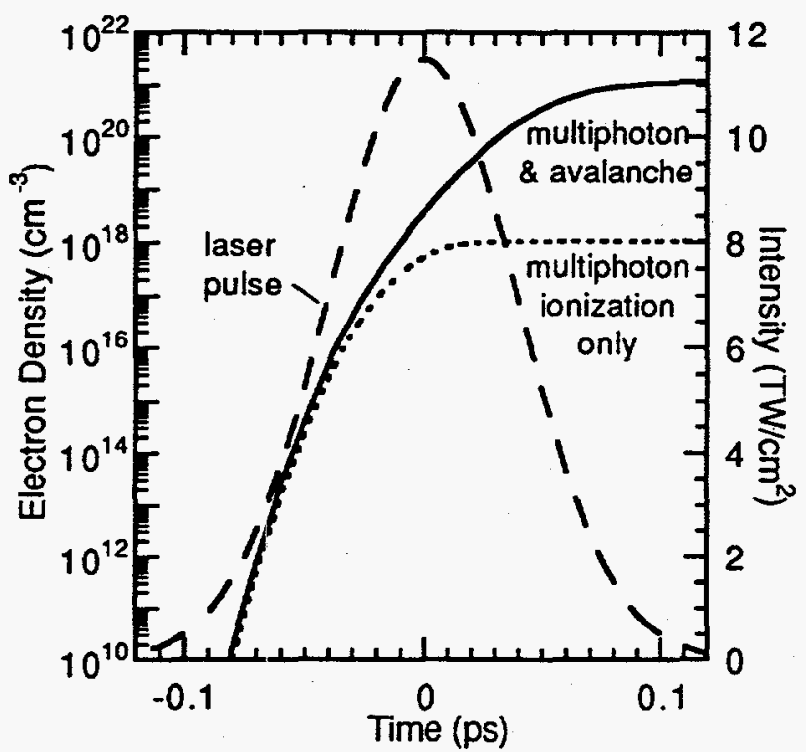

Figure 8. Total (upper solid) and multiphoton produced (lower solid) electron densities are plotted along with the Gaussian pulse shape. Seed electrons are produced by multiphoton ionization at the pulse peak after which an avalanche produces a critical density.

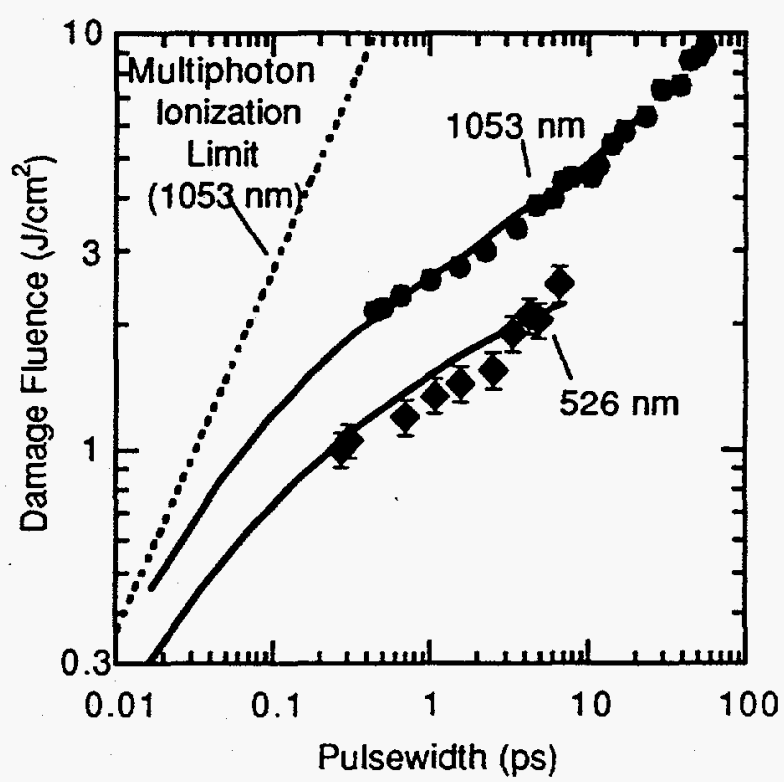

Figure 9. Measured and calculated (solid lines) damage fluence for fused silica at 1053 and $526 \mathrm{~nm}$. Dashed line indicates calculated damage limit due to multiphoton ionization alone. 
the peak of the pulse. After these "seed" electrons are produced, a small electron avalanche achieves a critical density plasma. It is important to note that the dense plasma is not produced until late in the pulse. Only this last part of the pulse experiences strong absorption or reflection. We expect thresholds to be more sensitive to the pulseshape for longer pulses, where the avalanche is relatively more significant.

In our modeling, we take the damage threshold to be indicated by the occurrence of a sufficiently high electron density. A reasonable lower limit would be on the order of $10^{19} \mathrm{~cm}^{-3}$, roughly the density at which the energy density of conduction electrons equals the binding energy of the lattice. A more realistic choice is the critical electron density, $\mathrm{n}_{\mathrm{cr}}$, at which the plasma becomes reflective $\left(10^{21} \mathrm{~cm}^{-3}\right.$ for 1053 $\mathrm{nm}$ ), since it is just below this density that the laser is strongly absorbed. Our calculations indicate the theoretical threshold is only logarithmically dependent on this choice.

In Figure 9, we compare our measured and calculated damage thresholds at $526 \mathrm{~nm}$ and $1053 \mathrm{~nm}$. The solid curves are the results of our theoretical modeling of laser-induced damage in the short-pulse limit, and are in very good agreement with both the pulsewidth and wavelength scaling of the measured data. As shown, with decreasing pulsewidth the damage threshold will asymptote to the limit where multiphoton ionization alone creates sufficient electron density to cause damage. At $1053 \mathrm{~nm}$ this asymptotic limit scales as $\tau^{7 / 8}$, and in general scales as $\tau^{(m-1) / m} n_{c r}^{1 / m}$ when m-photon ionization is the dominant process.

\subsection{Fluorides}

The damage threshold of calcium fluoride exhibits a similar pulsewidth dependence to that of fused silica (Figure 10). In the long-pulse limit, the threshold fluence also scales as $\tau^{1 / 2}$, and then changes to the short-pulse limit near $20 \mathrm{ps}$. For long pulses the damage morphology is again consistent with melting. Figure 11(a) shows the melting and recrystalization of the calcium fluoride surface layers, which occurred with no evidence of an avalanche breakdown. This is consistent with the measurements of Jones et al 15 on wide-gap alkali halides. Short-pulse damage clearly initiates on scratches left from the polishing process (Figure 11(b)), although as observed by Milam 31 with 125 ps pulses, the damage threshold did not appear to be greatly influenced by the polishing sleeks. The short-pulse $(0.4 \mathrm{ps})$ damage thresholds of $\mathrm{BaF}_{2}, \mathrm{CaF}_{2}, \mathrm{MgF}_{2}$, and $\mathrm{LiF}$ (included in Figure 14) scale with bandgap energy, as expected from multiphoton initiated avalanche ionization.

\subsection{Multilayer dielectrics}

We have tested several different multilayer dielectric mirrors and polarizers, with each multilayer stack consisting of approximately 20 individual layers of thickness $0.1-0.3 \mu \mathrm{m}$. Figure 12 shows the pulsewidth dependence of the 1053 -nm damage threshold fluence for a $45^{\circ}$ high-reflector and a $57^{\circ}$ polarizer used in reflection. The long-pulse scaling of each is slightly less than $\tau^{1 / 2}$, and again there is a transition to the short-pulse regime near $20 \mathrm{ps}$. The long-pulse damage morphology of the mirror is again characterized by melting and flow (Figure 13(a)), whereas short pulses cause ablation of the individual dielectric layers (Figure 13(b)). As has been thoroughly characterized by Kozlowski, et al ${ }^{32}$, the initiation of damage with long (ns) pulses is dominated by nodules and defects. We find the same behavior with short $(0.4 \mathrm{ps})$ pulses, where the presence of defects reduces the damage threshold by up to $50 \%$, depending on the size and type of defect. We tried laser-conditioning 33 the mirror to improve the short- 


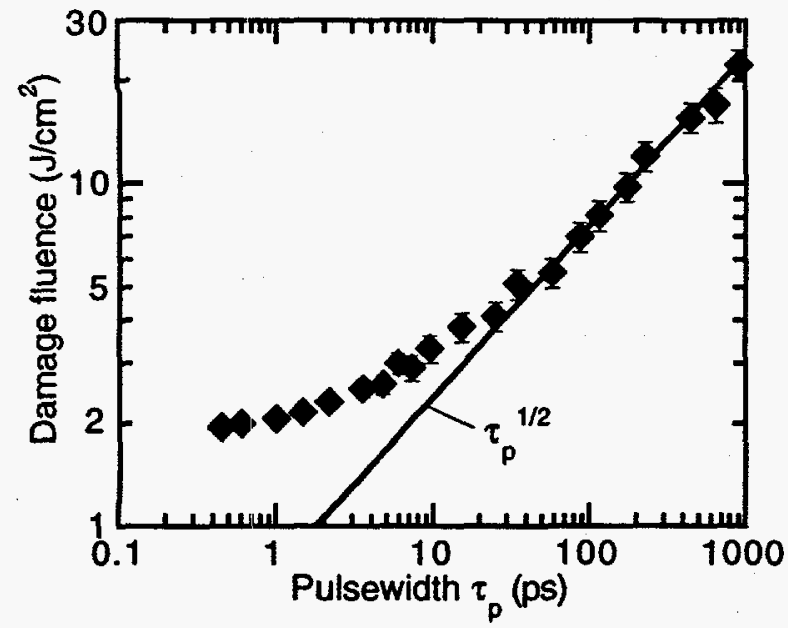

Figure 10. Pulsewidth dependence of threshold damage fluence for calcium fluoride.

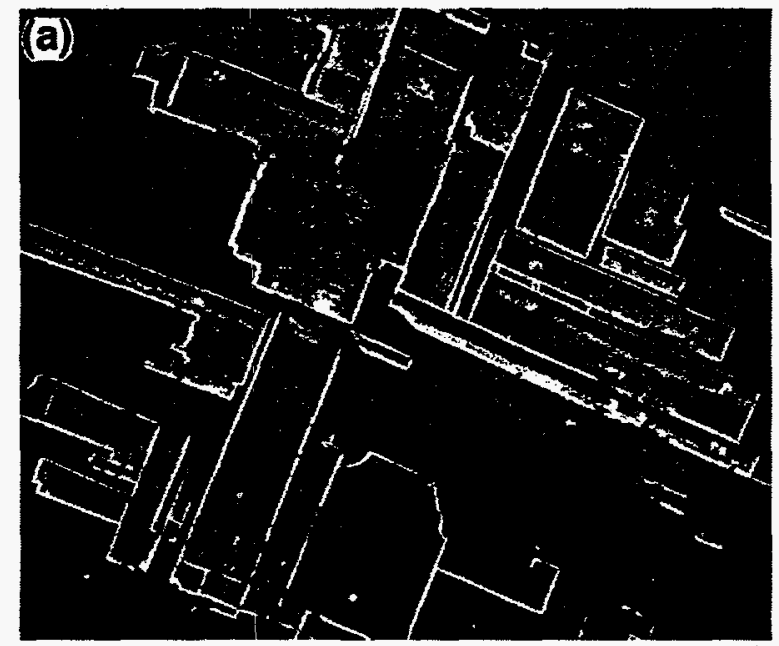

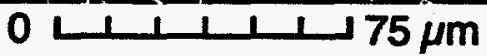

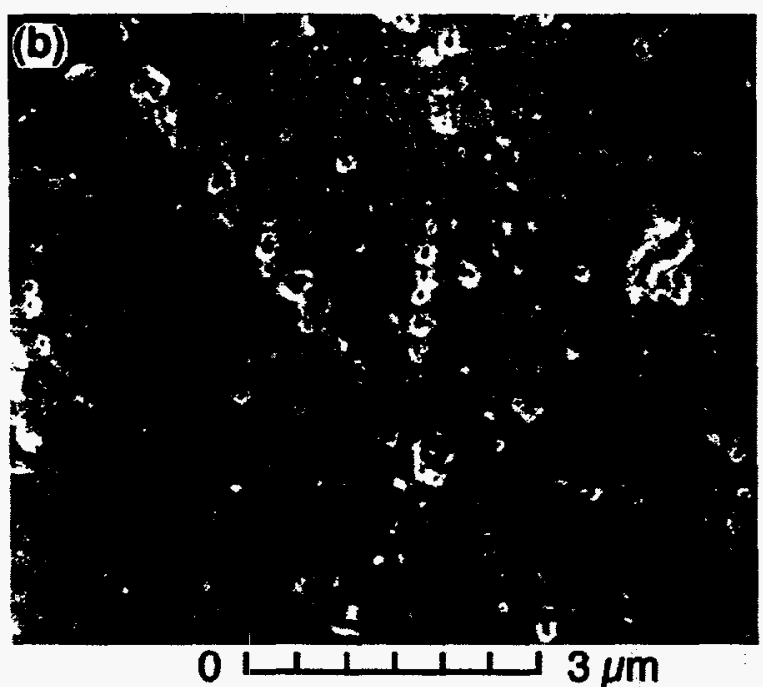

Figure 11. Laser damage morphology of calcium fluoride for (a) $900 \mathrm{ps}$, and (b) $0.4 \mathrm{ps}$ pulses.

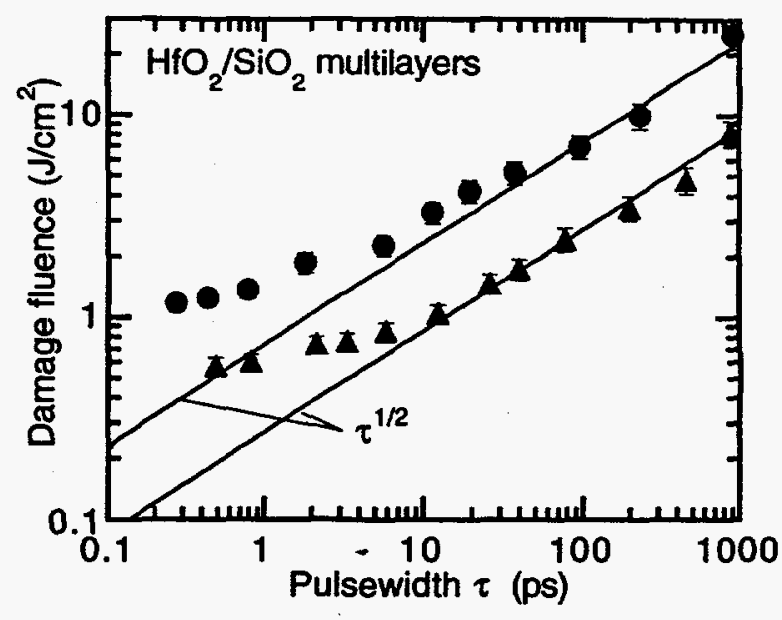

Figure 12. Pulsewidth dependence of threshold damage fluence for two different $\mathrm{HfO}_{2} / \mathrm{SiO}_{2}$ multilayer dielectric samples on relatively clean areas: $(\bullet) 57^{\circ} \mathrm{S}$ polarizer, $(\Delta)$ $45^{\circ} \mathrm{S}$ mirror.

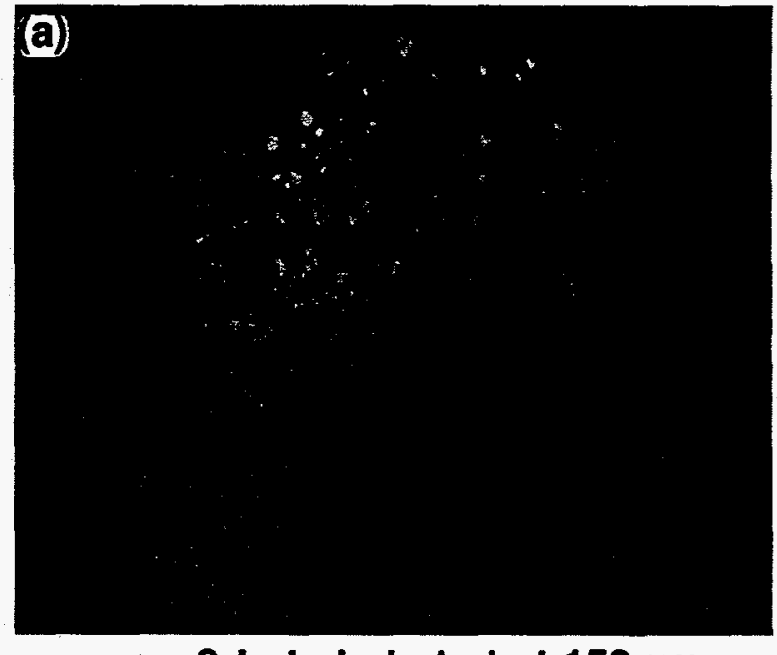

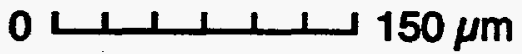

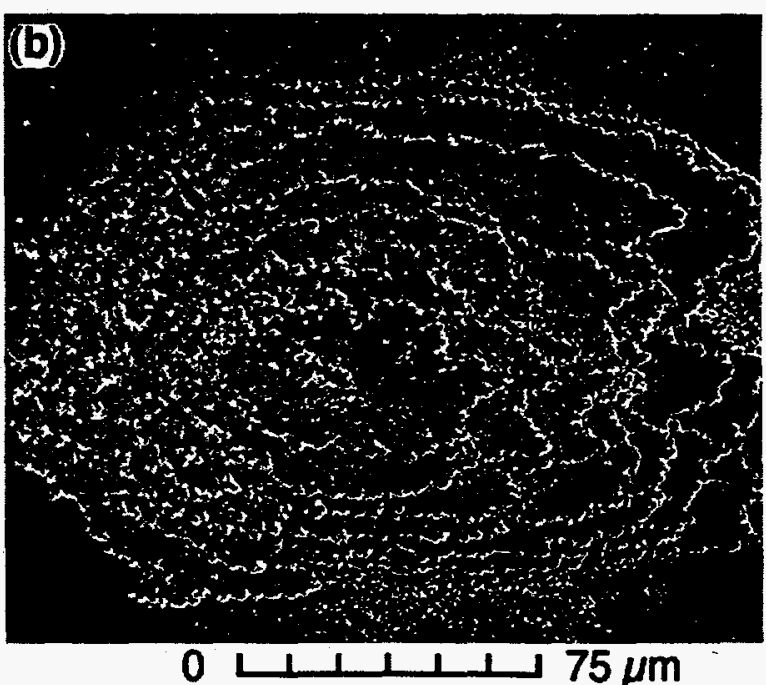

Figure 13. Laser damage morphology of a multilayer dielectric mirror for (a) $900 \mathrm{ps}$, and (b) $0.4 \mathrm{ps}$ pulses. 
pulse damage threshold. Unfortunately, neither long nor short-pulse conditioning resulted in a higher short-pulse damage threshold.

The short-pulse $(0.4 \mathrm{ps})$ damage thresholds of many of the multilayer dielectrics we tested are included in Figure 14. These samples consisted of a variety of mirrors and polarizers with either $\mathrm{HfO}_{2} / \mathrm{SiO}_{2}$ or $\mathrm{Ta}_{2} \mathrm{O}_{5} / \mathrm{SiO}_{2}$ multilayers deposited by e-beam evaporation or ion-beam sputtering. The cross-hatched area indicates thresholds achieved on "clean" areas, as opposed to areas with obvious defects. As with long pulses, we find the key to achieving increased short-pulse damage thresholds is the reduction in size and number density of the defects. The highest damage threshold we have found on any multilayer dielectric sample with 0.4 -ps pulses at $1053 \mathrm{~nm}$ is $1.4 \mathrm{~J} / \mathrm{cm}^{2}$. We expect that, with proper design and production, diffraction gratings based on these multilayer structures will exhibit damage thresholds comparable those of the multilayer itself.

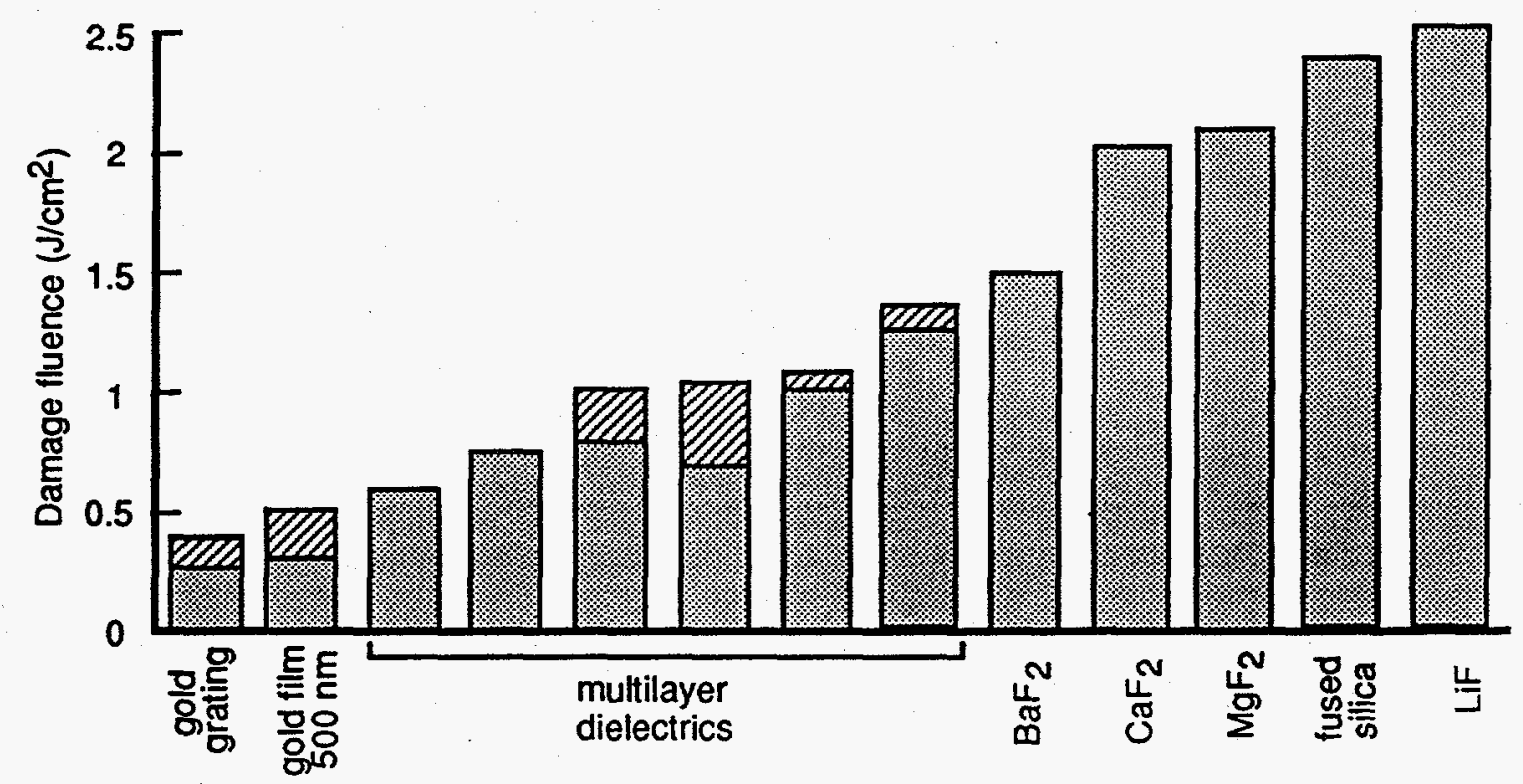

Figure 14. Short-pulse $(0.4 \mathrm{ps})$, front-surface threshold damage fluences for gold film, gold grating, multilayer dielectrics (polarizers and mirrors), and pure dielectrics.

\section{DISCUSSION}

We have investigated the pulsewidth dependence of laser-induced damage in pure and multilayer dielectrics and gold-coated optics over the range 0.1-1000 ps. The damage threshold of gold films increases with greater thickness for long (ns) pulses, where there is sufficient time for thermal diffusion. However, with subpicosecond pulses the damage threshold is limited to approximately $500 \mathrm{~mJ} / \mathrm{cm}^{2}$, and is independent of thickness.

In dielectrics, we observe a strong deviation from the long-pulse $\tau^{1 / 2}$ scaling of laser damage fluence for pulses shorter than $20 \mathrm{ps}$, below which electrons have insufficient time to couple to the lattice during the laser pulse. The damage threshold continues to decrease with decreasing pulsewidth, but at a rate slower than $\tau^{1 / 2}$ in the range 0.1 to $20 \mathrm{ps}$. This departure is accompanied by a qualitative change in the damage morphology indicative of rapid plasma formation and surface ablation. The damage site is limited to only a small region where the laser intensity is sufficient to produce a plasma with essentially no 
collateral damage. A theoretical model, in which initial electrons provided by multiphoton ionization are further heated resulting in collisional (avalanche) ionization, predicts short-pulse damage thresholds in excellent agreement with both the pulsewidth and wavelength scaling of our measurements. For extremely short pulses ( $\tau<30 \mathrm{fs}$ ), multiphoton ionization alone will provide the critical density of electrons.

\section{ACKNOWLEDGMENTS}

We would like to thank M. Kozlowski, F. Rainer, C. Stolz, R. Chow, I. Thomas, J. Britten, F. De Marco, J. Campbell, and L.J. Atherton for advice, equipment, and samples, and E. Lindsay for assistance with electron microscopy. This work was performed under the auspices of the U.S. Department of Energy by Lawrence Livermore National Laboratory under contract No. W-7405-ENG-48.

\section{REFERENCES}

1. D. Strickland and G. Mourou, "Compression of Amplified Chirped Optical Pulses", Opt. Comm. 56, 219-221, 1985; P. Maine, D. Strickland, P. Bado, M. Pessot, and G. Mourou, "Generation of Ultrahigh Peak Power Pulses by Chirped Pulse Amplification", IEEE J. Quantum Electron. 24, 398-403, 1988.

2. M.D. Perry and G. Mourou, "Terawatt to Petawatt Subpicosecond Lasers", Science 264, 917-924 1994.

3. M.D. Perry, J. Britten, C. Shannon, and E. Shults, U.S. Patent Pending.

4. E.S. Bliss, "Pulse Duration Dependence of Laser Damage Mechanisms", Opto-Electron. 3, 99-108, 1971.

5. N. Bloembergen, "Laser-Induced Breakdown in Solids", IEEE J. Quantum Electron. QE-10, 375-386, 1974.

6. L.H. Holway Jr. and D.W. Fradin, "Electron avalanche breakdown by laser radiation in insulating crystals", J. Appl. Phys. 46, 279-291, 1975.

7. A.S. Epifanov, A.A. Manenkov, and, A.M. Prokhorov, "Theory of avalanche ionization induced in transparent dielectrics by an electromagnetic field", Sov. Phys. JETP 43, 377-382, 1976.

8. W.L. Smith, J.H. Bechtel, and N. Bloembergen, "Picosecond laser-induced breakdown at 5321 and $3547 \AA$ : Observation of frequency-dependent behavior", Phys. Rev. B 15, 4039-4055, 1977.

9. A. Schmid, P. Kelly, P. Bräunlich, "Optical breakdown in alkali halides", Phys. Rev. B 16, 4569-4582, 1977.

10. B.G. Gorshkov, A.S. Epifanov, and A.A. Manenkov, "Avalanche ionization produced in solids by large radiation quanta and relative role of multiphoton ionization in laser-induced breakdown", Sov. Phys. JETP 49, 309- 315, 1979.

11. A. Vaidyanathan, T.W. Walker, and A.H. Guenther, "The Relative Roles of Avalanche Multiplication and Multiphoton Absorption in Laser-Induced Damage of Dielectrics", IEEE J. Quantum Electron. QE-16, 89-93, 1980.

12. M. Sparks, D. L. Mills, R. Warren, T. Holstein, A. A. Maradudin, L. J. Sham, E. Loh Jr., and D. F. King, "Theory of electron-avalanche breakdown in solids", Phys. Rev. B 24, 3519-3536, 1981.

13. R.M. Wood, Laser Damage in Optical Materials , Hilger, Boston, 1986.

14. A.A. Manenkov and A.M. Prokhorov, "Laser-induced damage in solids",Sov. Phys. Usp. 29, 104-122, 1986.

15. S.C. Jones, P. Braunlich, R.T. Casper, X.-A. Shen, and P. Kelly, "Recent progress on laser-induced modifications and intrinsic bulk damage of wide-gap optical materials", Opt. Eng. 28, 1039, 1989.

16. E.S. Bliss, D. Milam, and R.A. Bradbury, "Dielectric Mirror Damage by Laser Radiation over a Range of Pulse Durations and Beam Radii", Appl. Opt. 12, 677-689, 1973.

17. E.W. Van Stryland, M.J. Soileau, A.L. Smirl, and W.E. Williams, "Pulse-width and focal-volume dependence of laserinduced breakdown", Phys. Rev. B 23, 2144-2151, 1981.

18. W.H. Lowdermilk and D. Milam, "Laser-Induced Surface and Coating Damage", IEEE J. Quantum Electron. QE-17, 1888-1903, 1981.

19. T.W. Walker, A.H. Guenther, and P.E. Nielsen, "Pulsed Laser-Induced Damage to Thin-Film Optical Coatings-Part I: Experimental", IEEE J. Quantum Electron. QE-17, 2041-2052, 1981.

20. S.R. Foltyn and L.J. Jolin, "Long-Range Pulselength Scaling of $351 \mathrm{~nm}$ Laser Damage Thresholds", Laser-Induced Damage in Optical Materials: 1986, H.E. Bennett, A.H. Guenther, D. Milam, and B.E. Newnam, Editors, NIST Spec. Pub. $752,336-343,1988$. 
21. J.H. Campbell, F. Rainer, M. Kozlowski, C.R. Wolfe, I. Thomas, and F. Milanovich, "Damage resistant optics for a mega-joule solid-state laser", Laser-Induced Damage in Optical Materials: 1990, H.E. Bennett, L.L. Chase, A.H. Guenther, B.E. Newnam, and MJ. Soileau, Editors, Proc. SPIE 1441, 444-456, 1991.

22. K. Mann, H. Gerhardt, G. Pfeifer, and R. Wolf, "Influence of the laser pulse length and shape on the damage threshold of UV optics", Laser-Induced Damage in Optical Materials: 1991, H.E. Bennett, L.L. Chase, A.H. Guenther, B.E. Newnam, and M.J. Soileau, Editors, Proc. SPIE 1624, 436-443, 1992.

23. S.V. Garnov, S.M. Klimentov, A.A. Said, and M.J. Soileau, "Laser Damage of HR, AR-coatings, Monolayers and Bare Surfaces at 1064 nm", Laser-Induced Damage in Optical Materials: 1992, H.E. Bennett, L.L. Chase, A.H. Guenther, B.E. Newnam, and MJ. Soileau, Editors, Proc. SPIE 1848, 162-181, 1993.

24. D. Du, X. Liu, G. Korn, J. Squier, and G. Mourou, "Laser-induced breakdown by impact ionization in $\mathrm{SiO}_{2}$ with pulse widths from 7 ns to 150 fs", Appl. Phys. Lett. 64, 3071-3073, 1994.

${ }^{25}$ B.C. Stuart, M.D. Feit, A.M. Rubenchik, B.W. Shore, and M.D. Perry, "Laser-induced damage in dielectrics with nanosecond to subpicosecond pulses", Phys. Rev. Lett., to be published, 1995; B.C. Stuart, M.D. Feit, S. Herman, A.M. Rubenchik, B.W. Shore, and M.D. Perry, "Nanosecond to femtosecond laser-induced breakdown in dielectrics", Phys. Rev. B, submitted, 1995.

26. B.C. Stuart, S. Herman, M.D. Perry, "Chirped-Pulse Amplification in Ti:Sapphire Beyond $1 \mu \mathrm{m} "$, IEEE J. Quantum Electron. QE-31, to be published, March 1995.

27. T. Ditmire, H. Nguyen, and M.D. Perry, "Design and performance of a multiterawatt Cr:LiSrAlF6 laser system", J. Opt. Soc. Am. B 11, 580-590, 1994.

28. R.D. Boyd, J.A. Britten, D.E. Decker, B.W. Shore, B.C. Stuart, and M.D. Perry, "High-effiency metallic diffraction grating for laser applications", Appl. Opt., to be published, 1995.

29. E. Popov, L. Tsonev and D. Maystre, "Gratings-general properties of the Littrow mounting and energy flow distribution" J. Mod. Opt. 37, 367-377, 1990; E. Popov, L. Tsonev and D. Maystre, "Losses of plasmon surface waves on metallic grating" J. Mod. Opt. 37, 379-387, 1990; E. Popov and L. Tsonev, "Total absorption of light by metallic gratings and energy flow distribution" Surface Science 230, 290-294, 1990.

30. Y.P. Raizer, "Breakdown and heating of gases under the influence of a laser beam", Sov. Phys. Usp. 8, 650-673, 1966.

31. D. Milam, "Laser-induced damage at $1064 \mathrm{~nm}, 125 \mathrm{psec}$ ", Appl. Opt. 16, 1204-1213, 1977.

32. M.R. Kozlowski and R. Chow, "The role of defects in laser damage of multilayer coatings", Laser-Induced Damage in Optical Materials: 1993, H.E. Bennett, L.L. Chase, A.H. Guenther, B.E. Newnam, and M.J. Soileau, Editors, Proc. SPIE 2114, 640-649, 1994.

33. M.R. Kozlowski, M. Staggs, F. Rainer, and J.H. Stathis, "Laser Conditioning and Electronic Defect Measurements of $\mathrm{HfO}_{2}$ and $\mathrm{SiO}_{2}$ Thin Films", Laser-Induced Damage in Optical Materials: 1990, H.E. Bennett, L.L. Chase, A.H. Guenther, B.E. Newnam, and M.J. Soileau, Editors, Proc. SPIE 1441, 269-282, 1991. 